\title{
Comparison of Peak Expiratory Flow Rates (PEFR) between obese and non-obese Females
}

\author{
Shanmugapriya Chinnaiyan ${ }^{1, A-F \oplus}$, Vinodha Ramayyan ${ }^{1, A, C, E-F} \oplus$ \\ ${ }^{1}$ Thanjavur Medical College, India \\ A - Research concept and design, B - Collection and/or assembly of data, C - Data analysis and interpretation, \\ $D$ - Writing the article, E - Critical revision of the article, F - Final approval of the article
}

\begin{abstract}
Shanmugapriya C, Vinodha R. Comparison of Peak Expiratory Flow Rates (PEFR) between Obese and Nonobese Females. J Pre-Clin Clin Res. 2021; 15(3): 111-115. doi: 10.26444/jpccr/139199
\end{abstract}

\begin{abstract} aged 35-45. Students Unpaired t- test. significant at $p<0.01$ level.

\section{Key words}

BMI, PEFR, Peak Flow Meter, obesity
\end{abstract}

Introduction. In India, obesity is rapidly escalating in all age groups. Obesity has significant effects on respiratory function and reduces lung volume. Peak Expiratory Flow Rate (PEFR) demonstrates the calibre of the airways and is accepted worldwide as the objective indicator of ventilatory capacity, and is useful for the diagnosis and management of respiratory illness.

Objective. The aim of this study was to compare the Peak Expiratory Flow Rates between obese and non-obese females

Materials and method. 40 healthy obese females with $\mathrm{BMI} \geq 30 \mathrm{Kg} / \mathrm{m}^{2}$ and 40 healthy non-obese females with BMI (18.5$24.9 \mathrm{Kg} / \mathrm{m}^{2}$ ) as controls were included in the study. Subjects with H/o cardiopulmonary illness, asthma, diabetes mellitus, hypertension, smokers, on chronic medication, subjects who had noticeable weight gain / loss over the preceding 3 months, were all excluded. PEFR Measurements were performed using a Mini Wright's Peak Flow Meter. Three readings at 2 minutes intervals were recorded. The maximum of the 3 values were taken as the PEFR. The results were statistically analysed using

Results. PEFR in obese females (320 $\pm 28.06 \mathrm{~L} / \mathrm{Min})$ was significantly lower than the non-obese females (361 $\pm 29.17 \mathrm{~L} / \mathrm{Min})$, which was statistically significant $(p=0.000 ; p<0.05)$. PEFR was negatively correlated with BMI (Pearson's correlation $r=-.127$ )

Conclusions. There occurs a significant reduction in PEFR in obese females, compared to non-obese females. This study highlights the need for aggressive reduction of weight in obese females in order to increase respiratory efficiency.

\section{INTRODUCTION}

Nowadays, with the continued rise in standards of living, obesity is emerging as a global epidemic affecting not only developed nations but also the developing countries. This has been termed the 'New World Syndrome' that poses severe problems for the modern health industry [1].

Rapid urbanisation, industrialisation, globalisation, change in lifestyle and dietary habits, and consequent reduction in physical activity, have contributed to the increasing prevalence of obesity in urban areas. Energy-dense cheap food, labour-saving devices, motorised transports, and a sedentary life style have led to obesity. The modern lifestyle is a potent risk factor for obesity $[2,3]$.

The English word 'Obesity' is derived from the Latin word 'Obesus', meaning fat or plump [4]. Obesity is a complex disease arising from multifaceted interactions of genetic and environmental factors. Obesity is the abnormal or excess accumulation of fat resulting from an imbalance between energy intake and energy expenditure $[5,6]$. Obesity is more common among women with a similar BMI, and women may have more body fat than men. An excessive amount of body fat exerts an unfavourable burden not only on cardiac function, but also on lung functions by increasing the metabolic demands and work load of breathing. Obesity is an independent risk factor for cardiovascular morbidity and mortality [6]. The WHO

Address for correspondence: Vinodha Ramayyan, Thanjavur Medical College, 613004, Thanjavur, India

E-mail: mdphysiology@gmail.com

Received: 05.06.2021; accepted: 21.06.2021; first published: 29.06.2021 defines obesity as 'a condition with excessive fat accumulation in the body to the extent that the health and wellbeing are adversely affected' [7]. According to this WHO classification of obesity, based on the BMI (Body Mass Index), individuals with $\mathrm{BMI}>30 \mathrm{Kg} / \mathrm{m}^{2}$ are categorised as obese, $\mathrm{BMI}$ between $25-29.9 \mathrm{Kg} / \mathrm{m}^{2}$ as overweight and $\mathrm{BMI}>40 \mathrm{Kg} / \mathrm{m}^{2}$ as severe/ morbid obesity. As per Asian Indian guidelines, people are categorized as underweight $\left(<18.5 \mathrm{~kg} / \mathrm{m}^{2}\right)$, normal or lean BMI $\left(18.5-22.9 \mathrm{~kg} / \mathrm{m}^{2}\right)$, overweight $\left(23.0-24.9 \mathrm{~kg} / \mathrm{m}^{2}\right)$ and obese $\left(\geq 25 \mathrm{~kg} / \mathrm{m}^{2}\right)$ [7].

BMI is the method used to assess body fat content and is an indicator of obesity. Based on data on substantial morbidity, $\mathrm{BMI}>30 \mathrm{Kg} / \mathrm{m}^{2}$ is the commonly used threshold for obesity in both genders $[5,6]$. Weight and BMI are used as the measures of overall adiposity. Waist-hip ratio (WHR) and waist circumference (WC) are used as measures for abdominal obesity. Overall adiposity is considered as predictors of pulmonary functional status, whereas abdominal obesity is believed to influence the pulmonary function mechanically by changing lung compliance, the work of breathing and the elastic recoil $[8,9]$.

In India, the prevalence of obesity ranges from $10 \%-50 \%$ and morbid obesity affects $5 \%$ of the country's population. In recent years it has reached epidemic proportions among young females in whom it has become an increasingly important medical problem. More than $23 \%$ of women are either overweight or obese, which is higher than the prevalence among men (20\%). This is because predisposition to fat accumulation is a common occurrence in women as the sex hormones strongly influence adipocyte metabolism. 
The prevalence of obesity among Indian women has elevated by $10.6 \%$ to $12.6 \%$ during the past two decades [10]. Obesity is rapidly escalating in India in all age groups and exerts significant effects on respiratory function and reduces the lung volume. Increased thoracic and abdominal fat in obese individual increases the work of breathing and increases the respiratory insufficiency [11].

Pulmonary functions are generally determined by respiratory muscle strength, compliance of the thoracic cavity, airway resistance and elastic recoil of the lungs. Narrowing of airways is expressed in terms of various expiratory flow rates [8]. Peak Expiratory Flow Rate (PEFR) is a measure of airflow in the bronchial tree and indicates bronchial tone, and is affected by age, gender, height, body weight, and other physical activity. There is evidence that obesity has a link to bronchial hyper-responsiveness [12].

PEFR is one of the important parameters in pulmonary function testing that has evolved as a clinical tool for the diagnosis and prognosis of respiratory diseases due to airflow limitations, its severity and variations. PEFR is a simple, noninvasive conventional tool for measuring lung functions in field study, is a convenient tool for quantitative and qualitative estimation of pulmonary function. For the assessment of ventilatory capacity, PEFR is considered the simplest among the pulmonary function indices, first introduced by Adorn in 1942 as a measurement of ventilatory function, accepted in 1949 as an index of spirometry. It is defined as 'the largest expiratory flow rate achieved with a maximally forced effort from a position of maximal expiration, expressed in litres/ min.' The PEFR of healthy young Indian males and females is around 500 and $350 \mathrm{~L} / \mathrm{min}$, respectively [12, 13, 9, 10, 8].

Obese women without obvious respiratory illness have an increased risk of dyspnoea during exercise or during even a slight increase in physical activity. This can lead to early and easy fatiguability in the women leading to sedentary life style. Thus, there is need to find the effect of increased BMI on the PEFR in obese females compared to non-obese in order to adequately formulate an exercise prescription for improving functional capacity and weight reduction for obese females [10].

Obesity has myriad effects on pulmonary function. The respiratory rate is usually increased in order to compensate for the normally depressed tidal volumes. Total respiratory system compliance is decreased; lung volumes and especially Expiratory Reserve Volume (ERV), are the most consistently affected respiratory parameters. Oxygenation may be affected, probably as a consequence of microatelectasis at the lung bases $[8,9,10,11,12]$.

In individual patients, the distribution of fat may be more important than the BMI. High BMI has been reported to elevate pulmonary arterial pressure [14], and impaired respiratory function in obesity is associated with increased morbidity and mortality. At higher BMI, the number of patients with abnormal pulmonary function test (PFT) increased $[12,14,15]$. From this point of view, it is necessary to know the impact of obesity on respiratory parameters, and thereby to assess fitness in obese individuals using a simple non-invasive test which can be performed as an outpatient procedure. The outcome of this study may focus the need for the management of obesity with modification of life style as a potential therapeutic target.

\section{MATERIALS AND METHOD}

This study, for which 80 healthy females aged 35-45 years were selected, was undertaken at the Research Laboratory of the Department of Physiology during January to June 2018. Based on their BMI, they were divided into two groups - a study group and a control group. The study group consisted of 40 apparently healthy obese females with BMI $>30 \mathrm{Kg} / \mathrm{m}^{2}$ while the control group consisted of 40 healthy non-obese females with BMI between $18.5-24.9 \mathrm{Kg} / \mathrm{m}^{2}$.

Subjects under the age of 35 and over the age of 45 , subjects who were overweight $\left(\mathrm{BMI}=25-29.9 \mathrm{Kg} / \mathrm{m}^{2}\right)$, underweight $\left(\mathrm{BMI}<18.4 \mathrm{Kg} / \mathrm{m}^{2}\right)$, with diabetes mellitus, hypertension, cardiovascular diseases, previous history of Ischemic heart disease, COPD, any other pulmonary diseases, previous head injury (H/o), psychological illness, neurological illness, or on chronic medications were excluded from the study.

The study was approved by the Institutional Ethical Committee. Written informed consent was obtained from each subject and a detailed medical history was taken, and a thorough clinical examination performed to rule out any systemic illness. The weight of each subject was measured in kilograms by a standard weighing machine, and height was measured in centimetres by a Stadiometer. BMI was calculated using Quetelet's Index:

$$
\text { BMI }\left(\mathrm{Kg} / \mathrm{m}^{2}\right)=\text { Weight }(\mathrm{kg}) / \text { Height }\left(\mathrm{m}^{2}\right) \text {. }
$$

All the tests were performed in all the subjects in the morning between 09:00 - 11:00 and in thermo-neutral conditions. The subjects were allowed to acclimatise to the experimental and environmental conditions. The subjects were clearly informed about the test procedure and a trial of the procedure was also allowed for obtaining better performance during the study.

The subjects were asked to stand in an upright position with the Mini Wright's peak flow meter held horizontally in front of their mouth, they were then allowed to inhale deeply and close the lips firmly around the mouthpiece and with the nose clipped shut, to ensure that there were no air leaks around the lips. They were then told to exhale as hard as possible, and the number indicated by the cursor was noted. The procedure was repeated twice more, thereby obtaining three readings at an interval of two minutes. The maximum of the three values was taken as the PEFR.

All the datas were expressed as Mean \pm SD. The pooled data were subjected to statistical analysis. Student's unpaired t-test and Pearson's correlation coefficient test were used for statistical analysis; a $p$ value of $<0.05$ was accepted as significant.

\section{RESULTS}

The mean age of the subjects in the study group was $40.28 \pm 3.45$ yrs, and in the control group $-40.15 \pm 3.06$ yrs. Both groups were matched for age, gender and height. Weight and mean BMI were significantly higher $(\mathrm{p}=.000 ; \mathrm{p}<0.05)$ in the obese study group, compared to the non-obese control group (Tab. I).

Peak Expiratory Flow Rates were found to be significantly lower in obese women $(320 \pm 28.06 \mathrm{~L} / \mathrm{min})$, compared with the non-obese females $(361 \pm 29.17 \mathrm{~L} / \mathrm{min})$, which was statistically significant. $(\mathrm{p}=0.000 ; \mathrm{p}<0.05)($ Tab. 2). 
Table 1. Characteristics of the two groups

\begin{tabular}{lcc}
\hline Parameters & $\begin{array}{c}\text { Study Group } \\
(\text { Mean } \pm \text { SD }) \\
(\mathrm{n}=40)\end{array}$ & $\begin{array}{c}\text { Control Group } \\
(\text { Mean } \pm \text { SD }) \\
(\mathrm{n}=40)\end{array}$ \\
\hline Age $(\mathrm{Yrs})$ & $40.28 \pm 3.45$ & $40.15 \pm 3.06$ \\
\hline Height $(\mathrm{Cms})$ & $147.25 \pm 6.872$ & $150.7 \pm 6.398$ \\
\hline Weight $(\mathrm{Kg})$ & $70.95 \pm 10.667$ & $49.78 \pm 6.351$ \\
\hline BMI $\left(\mathrm{Kg} / \mathrm{m}^{2}\right)$ & $33.03 \pm 3.27$ & $21.94 \pm 1.973$
\end{tabular}

Table 2. PEFR comparison between obese and control group.

\begin{tabular}{cccc}
\hline PEFR(L/min) & Obese women & Nonobese women & $p$ Value \\
\cline { 2 - 4 } & $320 \pm 28.06$ & $361 \pm 29.17$ & ${ }^{*} .000(p<0.05)$ \\
\hline
\end{tabular}

(*p value $<0.05$ accepted as significant)

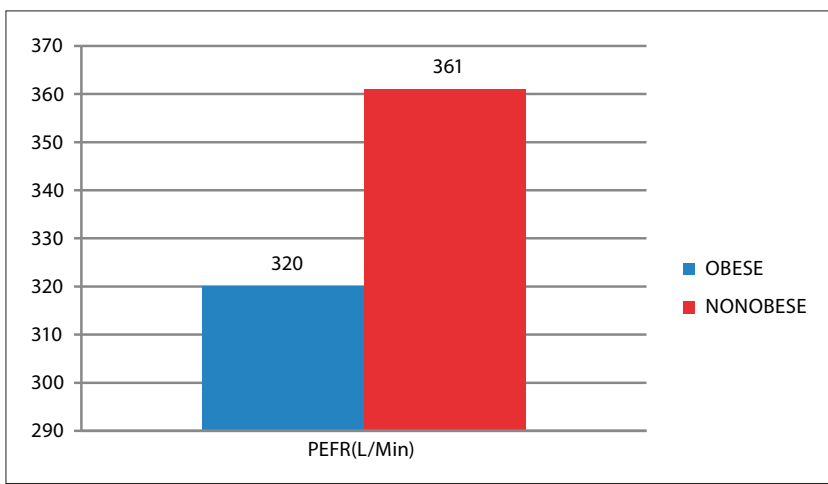

Figure 1. PEFR comparison between obese and non-obese females

Peak Expiratory Flow Rates were found to be negatively correlated with increasing BMI (Pearson's Correlation Coefficient: $\mathrm{r}=-.127)$ significant at $\mathrm{p}<0.01$ level (Tab. 3 and Fig. 2).

Table 3. Pearson's coefficient correlation between BMI and PEFR.

\begin{tabular}{lcc}
\hline BMI & Correlation value & Statistical inference \\
\hline PEFR & $-.127^{* *}$ & ${ }^{*} 000<0.01$ Significant \\
\hline
\end{tabular}

** Correlation significant at 0.01

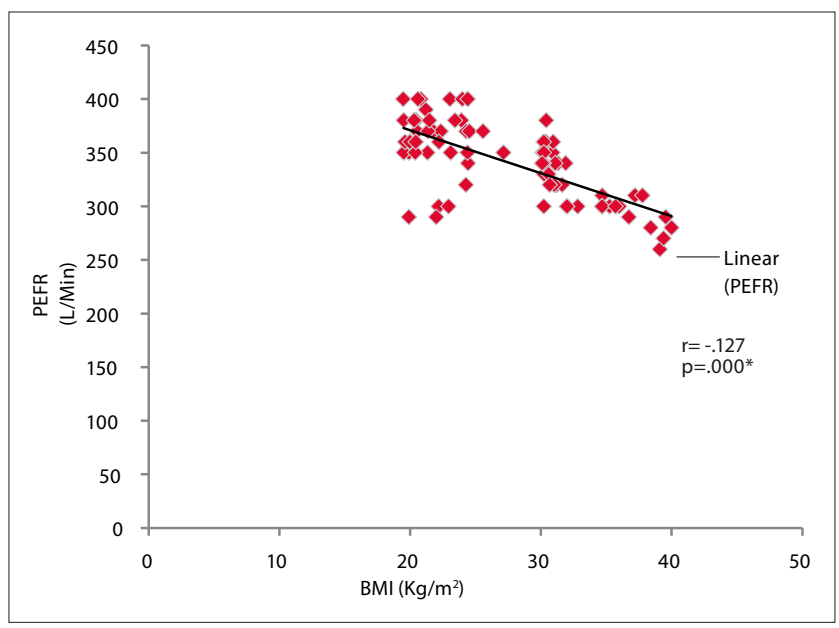

Figure 2. Pearson's coefficient of correlation between BMI and PEFR

\section{DISCUSSION}

PEFR measurement is a convenient tool for measuring lung functions in a field study and is fairly a good indicator of bronchial hyperresponsiveness. PEFR values are affected by various factors, such as gender, body surface area, obesity, posture, environmental and racial differences. Pulmonary functions are generally determined by respiratory muscle strength, compliance of the thoracic cavity, airway resistance, and elastic recoil of the lungs $[8,9,10,11,12,13,14,15]$.

The current study reported reduced PEFR in obese compared to non-obese females. Lower PEFR values in obese individuals in the study may be due to extra fat that exerted a mechanical effect on the movement of the chest or abdomen, or on the movement of the diaphragm, impeding descent into the abdominal cavity. This may also have been due to the fat deposition between the muscles and the ribs, leading to increase in the metabolic demands and work load of breathing. Obese individuals have an increased demand for ventilation and breathing work load, respiratory muscle insufficiency, decreased ERV, decreased FRC and closure of peripheral lung units. Obesity also influences upper airway tone and lung reflexes, lung mechanics may also affect the central control of breathing. Obesity adversely affects the chest wall mechanics and decreases the total respiratory compliance due to increased deposition of fat in subcutaneous adipose tissue. The literature also reveals lower PEFR values in obese compared to non-obese individuals. Joffa Paul Kwaku Price et al., in their study on correlation between BMI and PEFR in a Nigerian population, revealed a negative correlation between BMI and PEFR [13]. Nuba Kumar Das et al. correlated BMI and PEFR in brick industry labourers and found the results consistent with the presented study. They suggested that association of higher BMI with Low PEFR indicates that obesity is an important risk factor for reduced airflow and lung function, and they postulated that several possible mechanisms, such as mechanical effects on the diaphragm and fat deposition between muscles and ribs, can lead to an increase in the metabolic demands and workload of breathing, and consequently to reduced PEFR [16]. Basuli Gousami et al. hypothesized that PEFR is a consistent marker of respiratory illness associated with childhood obesity which can be evaluated from BMI/WHR. They reviewed a number of studies correlating pulmonary dysfunction and obesity, and hypothesized about the mechanical and biochemical aspects of reduced PEFR in obesity [12]. Yogesh Saxena et al. postulated that obesity itself and the pattern of body fat distribution have independent effects on PEFR, which strengthens the results of the presented study. They also suggested that abdominal obesity, measured from WHR, is a better predictor of expiratory flow than body weight and BMI, and that there is a significantly lower PEFR value in obese, compared to non-obese individuals [8]. Sunil Kumar Jena et al. in their research on relation of PEFR to BMI in young adults, showed a negative correlation between BMI with PEFR, which is consistent with the results obtained in the current study [17]. Sowmya Timmanna Koraddi et al. studied the effect of body fat distribution on young healthy obese students, and found that obesity and the pattern of fat distribution have independent effects on pulmonary function. This, too, supports the current study findings [18]. The hypothesis of the current study that obesity may be associated with significant reduction in PEFR was also 
substantiated by Saraswathy Ilango et al., who correlated obesity indices with PEFR and revealed that PEFR was significantly reduced in obese males [9]. Saylee R Patil et al. compared PEFR between obese and non-obese women and postulated that in the obese, the normal respiratory biomechanics are altered. Structural changes in the thoracoabdominal area, restricted diaphragmatic mobility and rib movements, promote changes in dynamics of the respiratory system and decreased respiratory compliance, leading to mechanical impairment of the respiratory muscles and decreased PEFR [10], which is designated as an objective indicator of pulmonary airflow resistance. The association of higher BMI with lower PEFR was also attributed to be a cue for greater risk factors correlated with childhood asthma [12].

Obesity has become a colossal epidemic causing serious concerns for public health. The increase in the prevalence of obesity is primarily due to the increasingly obesogenic environment rather than 'pathology' in metabolic defects or genetic mutations within individuals. In the studies showing the relationship between total mortality and obesity, it is seen that when BMI reaches above $30 \mathrm{Kg} / \mathrm{m}^{2}$, the incidence of mortality increases dramatically[19]. Laxmi et al concluded that BMI can be used in clinical settings to estimate body fat as it is a rapid and inexpensive method [20].

Visceral fat deposition negatively affects the pulmonary activities altering the air flow resistance, thereby altering the PEFR. This can be correlated with the onset of asthma, because obesity on the one hand can induce non-eosinophilic inflammatory pathways, increasing the risk of non-atopic asthma, or on the other hand, it can also affect the airway hyperresponsiveness (AHR). The overall adiposity compresses the chest wall, whereas the abdominal fat deposition causes respiratory muscle dysfunction [12].

An increasing level of obesity was found to significantly increase the total respiratory resistance and airway resistance in healthy obese subjects. Increased abdominal mass or abdominal adiposity restricts descent of the diaphragm into the abdominal cavity, which causes incomplete expansion of the chest and thus the lungs; therefore, increasing thoracic pressure. All these lead to the reduction of chest wall compliance, increased respiratory effort due to the added weight of breathing as the increased fat mass loads the respiratory apparatus that affects airway closure. All these lead to a negative impact on gaseous perfusion $[8,12]$.

Obesity increases oxygen consumption, which is attributed to the fact that increased fat deposition between the muscles and ribs might increase the metabolic demand. The increased thoracic and abdominal fats in obese subjects increase the work of breathing and decrease respiratory efficiency. In an attempt to reduce the work of breathing, there occurs a change in the breathing pattern to allow rapid respiration leading to increased respiratory rate[10].

All the above correlations between pulmonary dysfunction and obesity are from the mechanical aspect, whereas several studies have established an other probable association between these two - a biochemical association. Adipose tissue is an endocrine and energy storage organ composed of adipocytes, fibroblasts, endothelial cells, and immune cells. Adipose tissue acts as a source of pro-inflammatory cytokines and chemokines (Adipokines), such as IL-6, TNF- $\alpha$, leptin, IL-18 and Adiponectin, and acts by increasing their circulating levels or local concentration which leads to systemic inflammation and leaves a negative impact on pulmonary function. Under physiological and pathological conditions, adipokines induce the production of reactive oxygen species, which trigger oxidative stress. During this process, immune cells produce free oxygen radicals that promote a systemic pro-inflammatory state. These effects favour the development of bronchial hyperresponsiveness even in non-asthmatic individuals. This hyperresponsiveness of the airway due to its underlying inflammatory nature can compromise the size of the airway, leading to altered lung functioning. Thus, the main bridge is 'pulmonary inflammation' linking the visceral obesity and pulmonary function, which is also the key to asthma pathophysiology[17].

Hence, the conclusion was arrived at that altered mechanical muscular activity due to adiposity, altered airway calibre and increased respiratory resistance, may be responsible for the reduced lung functioning and lower PEFR in obese women. Young individuals with overweight and obesity, habituated to sedentary life style, are at greater risk for respiratory problems and prone to developing chronic obstructive pulmonary diseases at the later stage. PEFR, a consistent marker of respiratory illness and a convenient tool for quantitative and qualitative respiratory function, seems to be reduced in obesity.

The current study, although not exhaustive, is an attempt to bring awareness about change in lung function with increase in BMI by comparing PEFR in obese and non-obese females. This will assist in acknowledging the pulmonary health risks associated with an increasing BMI and fat accumulation. Therefore, reduced body weight achieved by aerobic exercise and a modified lifestyle will provide a significant improvement in PEFR in obese females.

\section{CONCLUSIONS}

A significant reduction in PEFR was noted in obese females compared to non-obese females. This may have been due to increased thoracic and abdominal fat in the obese females which increases the work of breathing and increases the respiratory insufficiency.

This study highlights the need for aggressive reduction of weight in obese individuals and there is scope for a population-based epidemiological study with a bigger sample size to further validate the findings of the study.

\section{Limitations of the study}

1. The study provided only a glimpse of the variety of alterations in respiratory systems, and further studies are needed to explore the underlying pathology in detail.

2 . This study included only obese females aged $35-45$, and did not include males. More studies are needed involving males as well as females of different age groups, and involving a larger sample size.

3. Obesity designated as $\mathrm{BMI}>30 \mathrm{~kg} / \mathrm{m}^{2}$ which indicates overall adiposity is used in this study. Instead, Waist Hip Ratio(WHR) which is a more reliable marker of abdominal obesity can be done in further epidemiological studies.

\section{Acknowledgement}

The authors express their thanks to all the participants and the technical personnel involved in this study. 


\section{Funding and conflicts of interest}

This research did not receive any specific grant from funding agencies in the public, commercial, or not-for-profit sectors. The authors and planners have disclosed no potential conflicts of interest, financial or otherwise.

\section{REFERENCES}

1. Ramezankhany A, Nazar Ali P, Hedayati M. Comparing Effects of Aerobics, Pilates Exercises and Low Calorie Diet on Leptin Levels and Lipid Profiles in Sedentary Women. Iranian Journal of Basic Medical Sciences. 2011; 14(3): 256-263. doi: 10.22038/ijbms.2011.5003

2. Geissler C, Powers H. Human Nutrition. 12th ed. Edinburg: Elsevier Saunders; 2011. p. 401-423.

3. Mengistie AB, Reddy RC, SyamBabu M. The effects of combined aerobic and resistance exercise training on obese adults, Northwest Ethiopia. Res J Recent Sci. 2013; 2(1): 59-66.

4. Rudalee Husale, Abhijit Diwate. Effect of obesity on PEFR value. VIMS J Physical Th. 2019; 1(2): 111-116.

5. Hall JE, Guyton AC. Textbook of Medical Physiology. 12th ed. New York: Elsevier Saunders; 2011. p. 850.

6. Longo DL, Fauci AS, Kasper DL, et al. Harrison's principles of Internal Medicine. 18th ed. New York: Mc Graw Hill; 2011. p. 622-633.

7. Runge MS, Greganti MA. Netter's Internal Medicine. 2nd ed. Elsevier Saunders; 2008. p 18-28.

8. Saxena Y, Purwar B, Upmanyu R. Adiposity: determinant of peak expiratory flow rate in young Indian adults male. Indian J Chest Dis Allied Sci. 2011; Jan-Mar; 53(1): 29-33. PMID: 21446222.

9. Saraswathi Ilango, Christy A, Saravanan A, Dr. Prema Sembulingam. Correlation of Obesity Indices with Peak Expiratory Flow Rate in Males and Females. IOSR Journal Of Pharmacy. 2014; 4(2): 21-27.
10. Patil SR, Mehta A. Comparison of peak expiratory flow rate in obese and non-obese women. Int J Health Sci Res. 2019; 9(9): 39-45.

11. Sarwari KN, Imtiaz Ali, Jaleeli KA, Shanmukhappa NJ. Assessment of pulmonary functions in young obese males and females in the age group 18-25 years. International Journal of Basic and Applied Medical Sciences. 2012; 2(3): 185-189.

12. Basuli Goswami, Anindita Singha Roy, Rishna Dalui and Amit Bandyopadhyay, Peak Expiratory Flow Rate - A Consistent Marker of Respiratory Illness Associated with Childhood Obesity. American Journal of Sports Science and Medicine. 2014; 2(1): 21-26. doi: 10.12691/ ajssm-2-1-4

13. Price JPK, Arthur N, Macstephen AO. Correlation between Body Mass Index and Peak Expiratory Flow Rate of an Indigenous Nigerian Population in the Niger Delta Region. Res J Recent Sci. 2013; 2(2): 28-32.

14. Pradeep Prajapatil, Neelima Singh1, Raj Kishori Prajapati2, Jagat Pal Singh. A prospective study of pulmonary function test in obese patient. Int J Adv Med. 2016; 3(1): 73-76.

15. Rudalee Husale, Abhijit Diwate. Effect of obesity on PEFR value.VIMS J Physical Th. 2019; 1(2): 111-116.

16. Das, Naba \& Sarkar, Gautam \& Khan, Ansar. Correlation Between Body Mass Index and Peak Expiratory Flow Rate of an Indigenous Brick Industry Labour on Bank of Kangsabati River, Paschim Medinipur, West Bengal. Indian Journal of Research. 2014; 3: 129-131.

17. Jena SK, Mirdha M, Meher P, Misra AK. Relation of peak expiratory flow rate to body mass index in young adults. Muller J Med Sci Res 2017; 8: 19-23.

18. Koraddi ST, Shrilaxmi Bagali, Manjunatha Aithala. Effect of Body Fat Distribution on Pulmonary Functions in Young Healthy Obese Students. JKIMSU. 2015; 4(4): 18-26.

19. Ajay KT, Vatsala AR, Suresh Y Bondade, Sonam D, Sangam. Effect of obesity on cardiovascular functions in adolescent male. J Pharm. Sci\&Res. 2014; 6(3): 164-166.

20. Laxmi CC, Udaya IB, Vinutha Shankar S. Effect of body mass index on cardiorespiratory fitness in young healthy males. International Journal of Scientific and Research Publications. 2014; 4(2): 1-3. 